\title{
Conhecimentos e comportamentos de acadêmicos de Medicina de uma instituição privada de Teresina frente a infecções sexualmente transmissíveis
}

\author{
Knowledge and conduct of Medical students from a private institution in Teresina facing sexually
} transmitted infections

Conocimientos e comportamientos de estudiantes de Medicina de una institución privada de teresina frente las infecciones sexualmente transmissibles

\section{Resumo}

Ingressar no curso de medicina traz aos acadêmicos a responsabilidade de obter conhecimentos para o autocuidado, orientação e conduta de pacientes sobre as IST (Infecções sexualmente transmissíveis). Este estudo teve como objetivo analisar o conhecimento objetivo de acadêmicos de medicina sobre trasmissão, manifestações, tratamento e prevenção de IST; analisar o comportamento sexual de acadêmicos de medicina em relação ao autocuidado de prevenção das IST e analisar a adesão de acadêmicos de medicina a formas de prevenção de IST. Trata-se de um estudo descritivo transversal quantitativo fundamentado em um questionário aplicado para 125 alunos. Os resultados evidenciaram, em relação ao conhecimento sobre IST, nível de informação adequado dos estudantes, e, em relação ao perfil epidemiológico, maior multiplicidade de parceiros sexuais no sexo masculino e maior taxa de vacinação satisfatória para HPV (Papiloma Vírus Humano) no sexo feminino. Os achados mostram um conhecimento satisfatório, e, epidemiologicamente, comportamentos de risco e má adesão a formas de prevenção dos acadêmicos frente as IST.

Palavras-chave: Infecções sexualmente transmissíveis; Educação médica; Saúde do estudante.

\begin{abstract}
Joining the medical course brings students the responsibility to acquire knowledge about self-care, enlightening and behavior of patients about STIs (Sexually Transmitted Infections). This study aimed to analyze the objective knowledge of medical students about the transmission, manifestations, treatment and prevention of STIs, analyze the sexual behavior of medical students in relation to self-care to prevent STIs and analyze the adherence of medical students to forms of STI prevention. This is a descriptive quantitative cross-sectional paper based on a questionnaire applied to 125 students. The results showed acceptable knowledge from the students, greater variety of sexual partners in males and a higher rate of satisfactory vaccination for HPV (Human Papilloma Virus) in females. The findings show satisfactory knowledge, risky behaviors and poor adherence to forms of prevention by students against STIs.
\end{abstract}

Keywords: Sexually transmitted infections; Medical education; Student health.

\section{Resumen}

Ingresar al curso de medicina trae a los estudiantes la responsabilidad de obtener conocimientos para el autocuidado, orientación y conducta de pacientes sobre las IST (Infecciones sexualmente transmisibles). Este estudio tuvo como objetivo analizar el conocimiento objetivo de los estudiantes de medicina sobre la transmisión, manifestaciones, tratamiento y prevención de las ITS, analizar el comportamiento sexual de los estudiantes de medicina en relación al autocuidado para prevenir las ITS y analizar la adherencia de los estudiantes de medicina a las formas de ITS prevención . Es un estudio cuantitativo transversal descriptivo basado en un cuestionario aplicado a 125 alumnos. Los resultados mostraron conocimiento adecuado de los estudiantes, mayor multiplicidad de parejas sexuales en los 
hombres y mayor tasa de vacunación para HPV (Papilomavirus humano) en las mujeres. Los achados muestran conocimientos satisfactorios, comportamientos de riesgo y mala adhesión a las formas de prevención por parte de los alunos frente las IST.

Palabras clave: Infecciones de transmisión sexual; Educación médica; Salud de los estudiantes.

\section{Introdução}

A partir do século XX, ocorreram muitas mudanças em todas as áreas do relacionamento e desenvolvimento humano, em especial, quanto à atividade produtiva, à organização do trabalho, aos processos educativos e de comunicação, até a socialização das novas gerações, ao universo de valores e critérios que orientam a conduta no cotidiano (Petrini, 2004). As revoluções não se limitaram à sociedade, mas também abrangem a visão da sexualidade que foi transformada no âmbito da cultura ocidental. Ao longo de muitos anos, notaram-se evoluções nas relações de gênero, introdução de métodos contraceptivos seguros e eficazes, a exemplo do dispositivo intrauterino de cobre ou de hormônio, cada vez mais popularizado, e um controle e tratamento mais eficiente das infecções sexualmente transmissíveis (IST), por meio da propagação de informação de prevenção e novas drogas introduzidas no mercado (Almeida et al., 2017).

A partir das mudanças na esfera dos comportamentos sexuais, por exemplo a iniciação precoce do ato sexual, e consequente desenvolvimento de comportamentos de risco, como o ato sexual desprotegido, associado ou não com o consumo de álcool, tabaco e outras drogas, houve impactos importantes sobre as IST (Lins, 2017). A importância da prevenção e conhecimento sobre essas infecções passou a ser mais valorizada a partir da emergência do HIV/AIDS na década de 1980. Assim, o uso da camisinha (preservativo de látex masculino) passou a ser o método preventivo mais divulgado por várias agências de saúde por seu elevado grau de proteção documentado frente ao risco de aquisição de infecções sexualmente transmissíveis (IST), inclusive o HIV/AIDS. Essas revoluções influenciam toda a sociedade, inclusive jovens universitários (Almeida et al., 2017; Guerra et al., 2020).

Com o ingresso no ensino superior, os jovens enfrentam novos desafios e se deparam com situações que moldam seus valores (Perim et al., 2020; Ramos et al., 2020). O ingresso em uma universidade traz à tona sensações e emoções que reforçam o conceito ilusório de autoreflexo de invulnerabilidade da juventude, e pode ser visto por meio de uma maior liberdade sexual que expõe os jovens a comportamentos de risco para a saúde e maiores riscos à aquisição de IST (Pimentel, 2016; Graf; Mesenburg; Fassa, 2020). Isso também se aplica a acadêmicos de medicina, que desde o início de suas práticas no curso, já começam a ter contato com pacientes (sobretudo na atenção básica); época que exige muita responsabilidade para obtenção de conhecimentos essenciais para a orientação de pacientes (Spindola et al., 2019).

Os jovens, em geral, possuem conhecimento sobre a importância do uso do preservativo para a prevenção de IST. No entanto, investigações têm demonstrado que a despeito dos avanços na área do conhecimento existem falhas no processo de prevenção, sendo um problema para a saúde pública brasileira modificar as atitudes e práticas preventivas do grupo jovem (Spindola et al., 2019; Fonte et al., 2018).

Este estudo teve como objetivo analisar o conhecimento de acadêmicos de medicina sobre trasmissão, manifestações e prevenção de IST, analisar o comportamento sexual de acadêmicos de medicina em relação ao autocuidado de prevenção das IST, visto que são informações fundamentais para a adequada prática médica; além de analisar a adesão de acadêmicos de medicina a formas de prevenção de IST. Por meio dessa análise, o estudo visa reunir informações que contribuam para uma melhor formação acadêmica e incentivar o emprego desse conhecimento em suas vidas pessoais e profissionais.

\section{Metodologia}

Trata-se de um estudo descritivo transversal com abordagem quantitativa, cuja coleta de dados foi realizada no período de 5 a 15 de outubro de 2020 no Centro Universitário Uninovafapi, em Teresina, Piauí. A coleta ocorreu após a 
aprovação pelo Comitê de Ética em Pesquisa do UNINOVAFAPI com o número do CAAE 36658920.8.0000.5210 em 28 de Agosto de 2020 em consonância com a Resolução 466/2012 do Conselho Nacional de Saúde e todas as resoluções vigentes no Brasil em relação à ética da pesquisa científica.O estudo contemplou 125 estudantes de medicina selecionados, aleatoriamente, entre os 184 , matriculados na UNINOVAFAPI, no $9^{\circ}, 10^{\circ}$ e $11^{\circ}$ períodos no segundo semestre de 2020 que aceitaram participar da pesquisa assinando o Termo de Consentimento Livre e Esclarecido (TCLE) e responderam o questionário completamente.

A abordagem dos alunos se deu em atividades referentes ao internato médico através da aplicação de um questionário anônimo que presou pela não identificação do aluno, para tanto, não houve campo para a identificação do acadêmico assim como todas as questões foram objetivas. Foi utilizado um código numérico em cada questionário como forma de distinção dos outros questionários.

O questionário, Quadro 1, apresentado abaixo, foi elaborado pelos pesquisadores e contém apenas questões objetivas. Neste, os acadêmicos foram inquiridos sobre sexo e faixa etária; conhecimento sobre IST, com quatro questões sobre transmissão, manifestações, possibilidade de cura definitiva e formas de prevenção; comportamento de risco, questionando se possui vida sexual ativa ou não, idade da primeira relação sexual, se possui parceiro fixo e se sim, há quanto tempo, número de parceiros desde a iniciação sexual e se faz uso de drogas ilícitas injetáveis com seringas compartilhadas; e adesão a formas de prevenção, questionando frequência do uso de preservativo nas suas relações sexuais, status de vacinação para hepatite B e HPV e quantas doses recebeu, se já fez algum exame de rastreio para IST e se já foi diagnosticado com alguma IST.

Quadro 1 - Questionário.

Código numérico:

1. Sexo: ( ) Masculino; ( ) Feminino
2. Qual a sua faixa etária?
( ) Entre 18 e 20 anos
( ) Entre 20 e 25 anos
( ) Entre 25 e 30 anos
( ) Acima de 30 anos

Avaliação do conhecimento sobre as IST

\title{
3. Selecione a alternativa que contém as formas mais prováveis de transmissão de infecção sexualmente transmissíveis.
}

( ) Contato de lesões ulceradas, picada de mosquito infectado, transmissão vertical.

( ) Uso de agulha não esterelizada para fazer tatuagens, relação sexual sem uso de preservativo, transmissão vertical.

( ) Uso compartilhado de alicate de unha em salão de beleza, Uso de vasos sanitários de banheiros públicos e compartilhamento de agulha para uso de drogas.

( ) Amamentação, picada de mosquito infectado, transmissão vertical

\author{
4. Selecione a alternativa que contém possível manifestação de IST causada por gonococos e clamídia. \\ ( ) Úlceras em região genital, \\ ( ) Úlceras orais. \\ ( ) Linfonodomegalia axilar. \\ ( ) Corrimento uretral masculino. \\ 5. Selecione a alternativa que contém APENAS IST que possuem cura DEFINITIVA: \\ ( ) Herpes, Gonorréia, Sifilis \\ ( ) HPV, Clamídia, Hepatite C \\ ( ) Gonorréia, Sifilis, Clamídia \\ ( ) Cancro mole, Hepatite C, Donovanose
}




\section{Selecione a opção que contém apenas métodos corretos de prevenção de IST: \\ ( ) Pílula anticoncepcional, Profilaxia Pré exposição(PREP), Condom. \\ ( ) Imunização para Hepatite B, Profilaxia pós exposição(PEP), Condom. \\ ( ) Condom, Profilaxia Pré exposição(PREP), DIU. \\ ( ) Diafragma, Imunização para Hepatite B, Profilaxia pós exposição(PEP).}

\section{Comportamento de risco para IST}

7. Você tem vida sexual ativa? ( ) Sim ; ( ) Não

Se sim, responda a questoes de 8 a 10

8. Idade da primeira relação sexual:

( ) Antes dos 12 anos

( ) 12 a 15 anos

( ) 16 a 19 anos

( ) 20 a 23 anos

( ) 24 a 27 anos

( ) Acima de 28 anos

9. Você tem parceiro fixo? ( ) Sim ; ( ) Não

9.1. Se sim, há quanto tempo?

( ) Menos de 6 meses

( ) Entre 6 meses e 1 ano

( ) Entre 1 ano e 2 anos

( ) Acima de 2 anos

10. Número de parceiros sexuais desde a iniciação sexual:

( ) 1

( ) 2 a 5

( ) 6 a 9

( ) 10 ou mais

11. Você já fez uso de drogas ilícitas injetáveis com seringas compartilhadas? （ ） Sim（） Não

\section{Adesão a formas de prevenção das IST}

12. Você faz uso do preservativo(camisinha) em suas relações sexuais? ( ) Sempre ( ) Ás vezes ( ) Nunca

Caso já teve relação sem o uso do preservativo(camisinha), responda as perguntas a seguir:

12.1. Você estava sobre a influência de álcool? ( )Sim; ( )Não

12.2. Você estava sobre a influência de drogas ilícitas? ( )Sim; ( )Não

12.3. Você o fez por medo de perder o/a parceiro(a) sexual caso se negasse? ( )Sim; ( )Não

12.4. Você o fez por estar sem preservativo no momento? ( )Sim; ( )Não

12.5. Você o fez por ser um/uma parceiro(a) fixo(a) e sentir-se seguro? ( )Sim; ( )Não

12.6. Você o fez por algum outro motivo não explicitado aqui? ( )Sim; ( )Não

13.Você já foi vacinado(a) para Hepatite B? ( )Sim ; ( ) Não

13.1 Se sim, quantas doses recebeu?

( ) Uma

( ) Duas

( ) Três

( ) Quatro ou mais.

14.Você já foi vacinado(a) para HPV? （ ) Sim ; ( ) Não

14.1.Se sim, quantas doses recebeu?

( ) Uma

( ) Duas

( ) Três 
15.Você já fez algum exame de rastreio para IST (HIV/Aids, hepatite B, hepatite C, sífilis, gonorreia, clamídia, HPV)? ( ) Sim; ( ) Não

16.Você já foi diagnosticado com alguma IST (HIV/Aids, hepatite B, hepatite C, sífilis, gonorreia, clamídia, HPV)?

( ) Sim; ( ) Não

Fonte: Dados da pesquisa

A análise estatística foi feita a partir de um estudo estatístico descritivo por meio das frequências absolutas (n) e relativas $(\%)$. O processamento dos dados foi realizado inicialmente através da planilha Excel® e, posteriormente, todos os dados foram processados e corrrelacionados no software Statistical Pack for the Social Science (SPSS)®, versão 20.0.

Para a análise univariada, foi utilizada a estatística descritiva através de gráficos e tabelas. Na bivariada utilizou-se o Teste G, conforme adequação. Foi considerado um nível de significância de $5 \%(p<0,05)$.

\section{Resultados e Discussão}

Dentre os 125 participantes, $69(55,2 \%)$ pertencem ao sexo masculino e $56(44,8 \%)$ ao sexo feminino. Em relação à faixa etária, $78(62,4 \%)$ têm entre 20 e 24 anos de idade , 40 (32\%) entre 25 e 29 anos e 7 (5,6\%) participantes acima de 30 anos.

Gráfico 1 - Percentual de acertos e erros a respeito das competências analisadas.

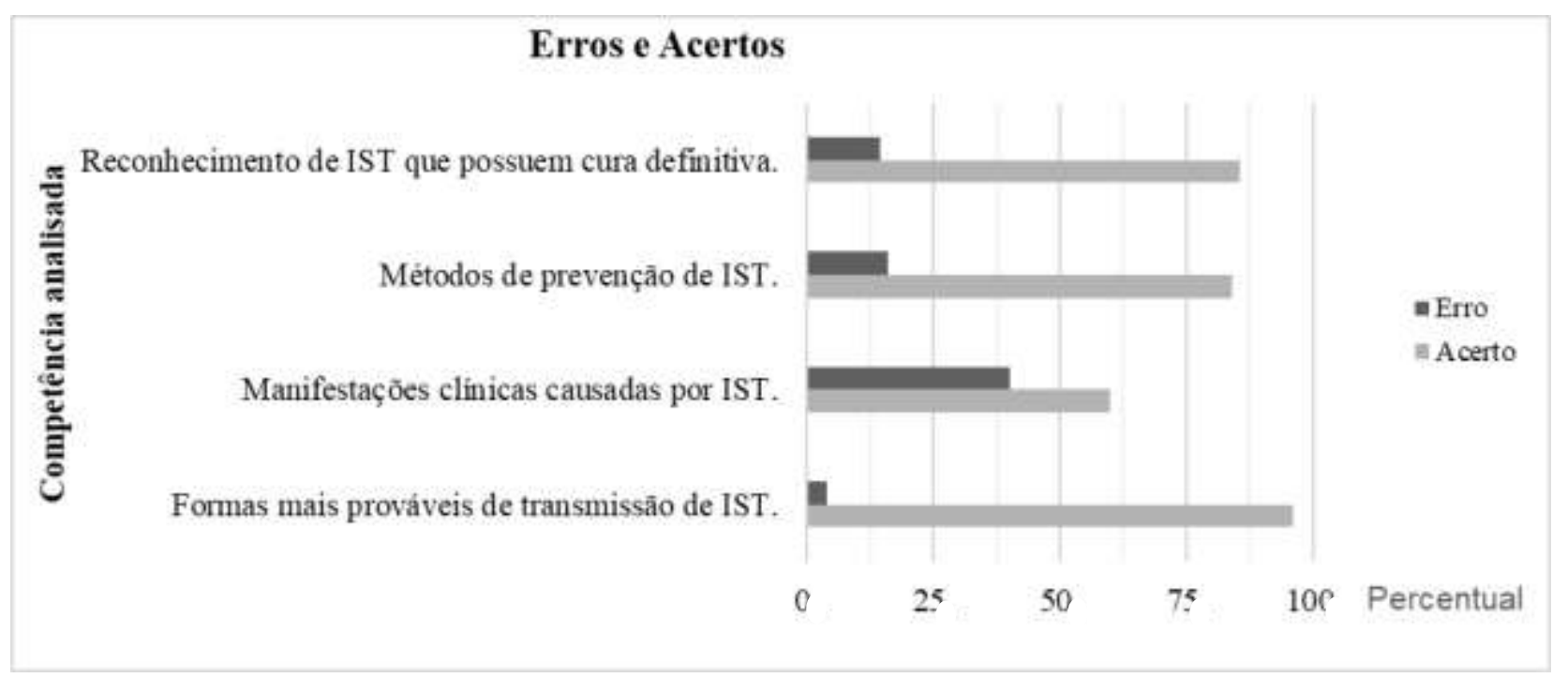

Fonte: Dados da pesquisa

No tocante à análise do conhecimento sobre as IST (Gráfico 1): a competência de conhecimento de métodos de prevenção dos participantes se mostrou excelente, já que atingiu uma taxa de $84 \%$ de acerto. O paciente que recebe as devidas orientações para prevenção de IST melhora a sua qualidade de vida e interrompe a cadeia de transmissão dessas infecções (Ministério da saúde do Brasil, 2015). Dessa forma, é importante o conhecimento da diversidade de formas de prevenção das IST, não somente para a vida pessoal, mas também para orientação adequada de pacientes (Ministério da saúde do Brasil, 2020).

A análise do reconhecimento de infecções sexualmente transmissíveis que possuem cura definitiva também se mostrou adequada. Na amostra analisada, alcançou-se uma taxa de $85,6 \%$ de acertos. Esse conhecimento se mostra 
imprescíndivel, já que em algumas IST, como por exemplo, o HIV/AIDS, não há um tratamento curativo definitivo (Lima, 2018).

Por outro lado, a análise das manifestações clínicas causadas por infecções sexualmente transmissíveis se mostrou precária quanto à taxa de acerto (60\%). Isso mostra que entre os acadêmicos analisados, há um deficit de conhecimento dessa competência. Portanto, isso pode acarretar um prejuízo no diagnóstico dos pacientes, haja vista que o reconhecimento de sinais e sintomas é o primeiro passo para o diagnóstico (Porto e porto, 2011).

Já a investigação do nível de conhecimento das formas de transmissão mais prováveis de IST se mostrou excelente em número de acertos (96\%). Isso mostra que os participantes dessa amostra possuem conhecimentos suficientes para orientar outras pessoas quanto aos riscos de transmissão de IST. Em consonância a isso, ao questionar estudantes de diversos cursos de uma universidade privada de Minas Gerais sobre formas de transmissão de HIV, sífilis, hepatite, gonorreia, herpes, HPV e clamídia, 100\% dos 154 entrevistados possuem conhecimento sobre a forma de transmissão vaginal e anal de IST, contudo, concluiu-se que os participantes mostraram falta de conhecimento quanto às formas de transmissão por via indireta. Além disso, vale ressaltar que as proporções relativas ao conhecimento sobre a possibilidade de contrair uma IST pelo contato com saliva, beijo e/ou uso de talheres apontaram que apenas 34 mulheres que possui este conhecimento $(32,1 \%)$, estatisticamente menor que o número de homens, 25 participantes, com o mesmo conhecimento (54,8\%) (Orlandi, 2021).

Quanto ao número de acertos nos questionamentos, 61 participantes (48,8\%) acertaram as quatro competências, 39 participantes $(31,2 \%)$ acertaram três questionamentos, 21 participantes (16,8\%) acertaram dois questionamentos, 4 participantes $(3,2 \%)$ acertaram 1 questionamento e nenhum participante errou todos os questionamentos. Isso se mostra satisfatório, já que $80 \%$ da amostra atingiu o conhecimento de pelo menos três questões. A quantidade de acertos também foi correlacionada com a faixa etária e o sexo dos acadêmicos. Contudo, a análise demonstrou que não existe significância estatística. Em comparação, ao questionar universitários de diversos cursos sobre formas de transmissão e possibilidade de cura de IST, Fonte e seus colaboradores concluíram que 313, apenas 40,75\% de seus participantes, obtiveram resultado acima da média e nenhum participante acertou todos os questionamentos (Fonte, 2018).

Além disso, a partir da análise dos comportamentos de risco para IST dos 125 acadêmicos, foi possível constatar que $117(93,6 \%)$ possuem vida sexual ativa. Dentro desse universo, 23 (19,7\%) relataram início da vida sexual com idade igual ou inferior aos 15 anos, 66 (56,4\%) entre 16 e 19 anos, 23 (19,7\%) entre 20 e 23 anos e, por fim, 5 (4,3\%) acima de 24 anos.

Dentro da amostra dos participantes que possuem vida sexual ativa, foi questionado se os participantes possuem parceiros fixos. Assim, $84(71,8 \%)$ responderam que sim e 33 (28,2\%) responderam que não. A partir dos participantes que declararam ter parceiros fixos, $9(10,7 \%)$ declararam ter parceiro fixo a menos de 6 meses, $9(10,7 \%)$ entre 6 meses e 1 ano, 8 $(9,6 \%)$ entre 1 ano e 2 anos e 58 ( $69 \%)$, acima de 2 anos.

Tabela 1 - Número de parceiros sexuais durante a vida por sexo.

\begin{tabular}{c|c|c|c|c|c|c}
\hline \multicolumn{2}{c}{} & \multicolumn{2}{l|}{ Sexo } & \\
\cline { 3 - 7 } & \multicolumn{2}{l|}{ Feminino } & \multicolumn{2}{l|}{ Masculino } & \\
\cline { 3 - 7 } & Frequência & $\%$ & Frequência & $\%$ & Valor de p \\
\hline \multirow{2}{*}{$\begin{array}{c}\text { Número de } \\
\text { parceiros sexuais } \\
\text { durante a vida }\end{array}$} & 2 a 9 & 6 & $4,8 \%$ & 2 & $1,6 \%$ & $\mathrm{p}<0,001$ \\
& 10 ou mais & 39 & $19,2 \%$ & 5 & $4,0 \%$ & \\
& 5 & $4,0 \%$ & 23 & $18,4 \%$ & \\
\hline
\end{tabular}


Ao correlacionar o número de parceiros sexuais durante a vida com o sexo dos participantes, notou-se significância estatística apontando que dentre os participantes que possuem 10 ou mais parceiros, o sexo masculino tem um número cinco vezes maior em relação ao sexo feminino, o que pode ser explicado por fatores socioculturais, e pela comunicação familiar maior diálogo e orientação parental com o sexo feminimo - que ratificam esse cenário (De Sales, 2020). Em consonância, dentre os participantes que possuem apenas 1 parceiro, o sexo feminino tem um número três vezes maior que o masculino. Essa informação sugere um padrão de comportamento de risco para o sexo masculino, visto que a multiplicidade de parceiros é considerada um fator de risco associado às IST. Em contrapartida, o número de parceiros sexuais durante a vida correlacionado à faixa etária não apresentou significância estatística (Henderson et al., 2020).

Dentre os 117 participantes que responderam ter vida sexual ativa, 25 (20\% do total de acadêmicos) informaram sempre usar preservativo durante a relação sexual, 83 (66,4\% do total de acadêmicos) informaram usar o preservativo às vezes e 9 (7,2\% do total de acadêmicos) informaram nunca usar preservativo. Logo, percebe-se que 73,6\% do total de participantes já teve relação sexual sem o uso do preservativo. Essa informação se mostra particularmente preocupante ao observar que a camisinha, tanto masculina quanto feminina, é o método mais conhecido, acessível e eficaz para prevenção de IST, entretanto, seu uso é ou já foi ignorado em alguma ocasião pela maioria dos participantes da pesquisa (Ministério da Saúde, 2020).

Tabela 2 - Fatores que influenciaram o não uso da camisinha.

\begin{tabular}{|c|c|c|c|}
\hline & & Frequência & $\%$ \\
\hline \multirow{2}{*}{ Influência de álcool para não uso da camisinha } & Não & 52 & $56,5 \%$ \\
\hline & Sim & 40 & $43,5 \%$ \\
\hline \multirow{2}{*}{ Influência de drogas ilícitas para não uso da camisinha } & Não & 81 & $88,0 \%$ \\
\hline & Sim & 11 & $12,0 \%$ \\
\hline \multirow{2}{*}{$\begin{array}{l}\text { Influência do medo de perder o/a parceiro(a) sexual caso se } \\
\text { negasse a usar camisinha }\end{array}$} & Não & 79 & $85,9 \%$ \\
\hline & Sim & 13 & $14,1 \%$ \\
\hline \multirow{2}{*}{$\begin{array}{l}\text { Participantes que relatam não adesão à camisinha por estar sem } \\
\text { preservativo no momento }\end{array}$} & Não & 42 & $45,7 \%$ \\
\hline & Sim & 50 & $54,3 \%$ \\
\hline \multirow{2}{*}{$\begin{array}{l}\text { Participantes que relatam não adesão ao uso da camisinha por } \\
\text { sentir-se seguro com um(a) parceiro(a) fixo(a) }\end{array}$} & Não & 11 & $12.0 \%$ \\
\hline & Sim & 81 & $88,0 \%$ \\
\hline \multirow{2}{*}{$\begin{array}{l}\text { Participantes que relatam não adesão ao uso da camisinha por } \\
\text { algum outro motivo não explicitado aqui }\end{array}$} & Não & 57 & $62,0 \%$ \\
\hline & Sim & 35 & $38,0 \%$ \\
\hline
\end{tabular}

Fonte: Dados da pesquisa

Conforme a Tabela 2, entre os participantes que já tiveram relação sexual sem o uso de camisinha, 43,5\% o fizeram sobre influência de álcool. Esse número se mostra bastante expressivo quando comparado a um estudo com resultados semelhantes realizado em Tubarão-SC, em que 18,9\% de uma amostra de acadêmicos de medicina declarou a não aderência ao uso da camisinha pelo mesmo motivo (Trevisol e Manoel, 2017). Ademais, 54,3\% da amostra já tiveram relação sexual sem camisinha por estar sem preservativo no momento.

Por fim, 88\% (81 acadêmicos) já tiveram relação sexual sem camisinha por sentir-se seguros com um parceiro fixo. Em consonância, ao coletar dados de 768 estudantes de uma universidade privada do Rio de Janeiro, Ramos e seus 
colaboradores concluíram que 55,9\% dos participantes que possuíam parceiro sexual fixo (285 estudantes) não faziam uso de preservativo durante a relação sexual. Esses dados se mostram preocupantes, pois podem sugerir uma falsa percepção de segurança entre os jovens que possuem relacionamento fixo acarretando um comportamento de risco para a aquisição de IST.

Os fatores motivadores para o não uso do preservativo foram correlacionados ao sexo e à faixa etária, porém não atingiram significância estatística. Em adição, 121 participantes (96,8\% do total de acadêmicos) informaram ter sido vacinados para hepatite B, destes, 72 (57,6\% do total de acadêmicos) receberam 3 ou mais doses estando satisfatoriamente vacinados de acordo com o esquema do Ministério da Saúde.

Em um estudo com resultados semelhantes, realizado com acadêmicos de Medicina de uma faculdade de Volta Redonda no Estado do Rio de Janeiro em 2018, 54\% dos alunos analisados ( distribuidos entre o $2^{\circ}, 6^{\circ}$, $9^{\circ}$ e $11^{\circ}$ semestres), declararam ter recebido 3 ou mais doses para hepatite B (Aragão et al., 2011). Esses dados se mostram alarmantes, tendo em vista que grande parte das doenças infecciosas transmitidas entre trabalhadores da área da saúde e acadêmicos está relacionada à exposição ocupacional, sobretudo, por acidentes com material perfurocortante. Haja vista que, esses acidentes expõem os individuos a microorganismos patogênicos, inclusive à hepatite B (Araújo; Silva, 2014).

A prevenção da hepatite B mais eficiente ocorre pela imunização, através de três doses da vacina. Essa vacina está disponível no serviço público de saúde e é iniciada a partir do nascimento. Em relação à vacina de HPV, 43 participantes (34,4\% do total de acadêmicos) informaram ter sido vacinados, dentre estes, 37 (29,6\% do total de acadêmicos) receberam duas ou mais doses, estando satisfatoriamente vacinados de acordo com o esquema do Ministério da Saúde. Para que sejam atingidos títulos de anticorpos satisfatoriamente protetores contra o HPV, faz-se necessária admnistração de, no mínimo, duas doses da vacina (Zardo et al, 2014; Rossi et al., 2021).

Tabela 3 - Relação entre esquema vacinal satisfatório ou insatisfatório para HPV e sexo dos entrevistados.

\begin{tabular}{c|c|c|c|c|c}
\hline \multirow{2}{*}{} & \multicolumn{4}{c|}{ Sexo } & \multirow{2}{*}{} \\
\cline { 2 - 5 } & \multicolumn{2}{|c|}{ Feminino } & \multicolumn{2}{c}{ Masculino } & \\
\cline { 2 - 5 } & Frequência & $\%$ & Frequência & $\%$ & Valor de p \\
\hline $\begin{array}{c}\text { Esquema vacinal } \\
\text { insatisfatório }\end{array}$ & 39 & $31,2 \%$ & 48 & $38,4 \%$ & 0,0004 \\
\hline $\begin{array}{c}\text { Esquema vacinal } \\
\text { satisfatório }\end{array}$ & 30 & $24,0 \%$ & 8 & $6,4 \%$ & \\
\hline
\end{tabular}

Teste $\mathrm{G}, \mathrm{p}<0,05$, existe correlação entre sexo e esquema vacinal satisfatório para HPV. Fonte: Dados da pesquisa;

A partir da correlação entre o esquema vacinal para Hepatite B e o sexo dos participantes, nota-se que não há significância estatística. Em contrapartida, nota-se que há significância entre o esquema vacinal para HPV e o sexo dos acadêmicos. É possivel notar uma prevalência maior de imunização satisfatória no sexo feminino, de quase quatro vezes maior, em relação ao sexo masculino.

Em comparação, uma pesquisa realizada com 379 participantes do $1^{\circ}$ ao $6^{\circ}$ ano do curso de medicina, em uma faculdade de Brasília, percebeu-se que a taxa de vacinação do sexo masculino foi um quarto da taxa do sexo feminino. Uma possível explicação para esse fato seria a feminização da vacina, atribuída à estratégia inicial de implantação da vacinação contra o HPV. Além disso, os estudantes do sexo masculino possivelmente se sentem mais protegidos das infecções pelo HPV (Wanderley, 2019). 
Tabela 4 - Relação entre esquema vacinal satisfatório ou insatisfatório para HPV e faixa etária dos entrevistados.

\begin{tabular}{c|c|c|c|c|c|c|c}
\hline \multirow{2}{*}{} & \multicolumn{5}{c|}{ Faixa Etária } & \multicolumn{2}{c}{} \\
\cline { 2 - 7 } & \multicolumn{2}{|c|}{ Acima de 30 anos } & \multicolumn{2}{c|}{ Entre 20 e 24 anos } & \multicolumn{2}{c}{ Entre 25 e 29 anos } & \\
\cline { 2 - 7 } & Frequência & $\%$ & Frequência & $\%$ & Frequência & $\%$ & Valor de p \\
\hline $\begin{array}{c}\text { Esquema vacinal } \\
\text { insatisfatório }\end{array}$ & 6 & $4,8 \%$ & 48 & $38,4 \%$ & 33 & $26,4 \%$ & 0,042 \\
\hline $\begin{array}{c}\text { Esquema vacinal } \\
\text { satisfatório }\end{array}$ & 1 & $0,8 \%$ & 30 & $24 \%$ & 7 & $5,6 \%$ & \\
\hline
\end{tabular}

Teste G, p < 0,05, existe correlação entre faixa etária e esquema vacinal satisfatório para HPV. Fonte: Dados da pesquisa.

A correlação entre o esquema vacinal satisfatório para Hepatite B e a faixa etária dos participantes não apontou significância estatística. Por outro lado, ao correlacionar os dados de esquema vacinal satisfatório para HPV com a faixa etária dos participantes obteve-se significância estatística demonstrando que a faixa etária de 20 a 25 anos concentrou o maior número de esquemas vacinais satisfatórios (Tabela 4). Uma possível explicação para esse resultado seria o fato de que a maioria dos estudantes não fazia parte da faixa etária alvo do programa de imunização nacional iniciado em 2014, necessitando, portanto, custear a vacinação (Wanderley et al, 2019).

Além disso, 94 participantes $(75,2 \%$ do total) informaram já ter realizado exame de rastreio para IST. Esses dados foram correlacionados com sexo e faixa etária dos participantes, porém não houve significância estatística. Quando questionados sobre diagnóstico prévio de IST, 11 participantes (8,8\% do total) afirmaram já ter recebido diagnóstico de IST. Essas informações foram correlacionadas com sexo e faixa etária dos participantes, porém não houve significância estatística.

Por fim, foram agrupados e contabilizados comportamentos e atitudes de cada participante que podem acarretar um maior risco para a aquisição de IST, como possuir 10 ou mais parceiros sexuais desde a iniciação sexual, ter relação sexual sem usar preservativo, não ter esquema vacinal satisfatório para Hepatite B, não ter esquema vacinal satisfatório para HPV e nunca ter realizado exame de rastreio para IST. Nesse contexto, observou-se que 23 participantes (18,4\% do total) apresentam 0 ou 1 comportamento e atitude de risco dos citados acima, 84 participantes (67,2\% do total) apresentam 2 ou 3 comportamentos e atitudes de risco e 18 participantes (14,4\% do total) apresentam 4 ou 5 comportamentos e atitudes de risco.

Logo, percebe-se que 102 participantes (81,6\% do total) possuem ao menos 2 comportamentos e atitudes de risco para contrair IST. Essa informação se torna particularmente preocupante ao ser comparada ao fato de que 100 participantes (80\% do total) acertaram ao menos três das quatro questões de conhecimento acerca de IST, sugerindo que os participantes possuem o conhecimento sobre forma de transmissão, manifestações, cura definitiva e métodos de prevenção de IST, porém não aplicam esse conhecimento em suas vidas pessoais.

\section{Considerações Finais}

Os achados da pesquisa evidenciam que a maioria dos universitários possui conhecimento suficiente em relação às IST, apesar do estudo evidenciar uma deficiência no reconhecimento de suas formas de manifestações clínicas. O que sugere a necessidade ampliação dos componentes curriculares que abordam a temática, a fim de suprir essa lacuna no conhecimento dos universitários e, consequentemente, formar profissionais competentes.

Observou-se maior comportamento de risco no sexo masculino em relação à maior multiplicidade de parceiros do sexo masculino e a tendência a um parceiro único do sexo feminino. Além disso, uma provável explicação para o não uso do 
preservativo por acadêmicos com um(a) parceiro(a) fixo(a) seria a falsa sensação de proteção ou segurança por se acreditar estar em um relacionamento monogâmico. Isso representa maior risco a essa população, já que a camisinha é o método de prevenção mais eficaz.

Em adição, a maior adesão a imunização para o HPV no sexo feminino e a preponderância da faixa etária de 20 a 25 anos podem ser explicadas por participarem dos grupos priorizados pelo Ministério da saúde para receber a vacina em campanhas anteriores de vacinação. Outra possível explicação seria a falsa percepção masculina da pouca repercussão do vírus HPV para a sua saúde. Considerando os resultados desse estudo, pode-se inferir que se faz necessário sensibilizar esse grupo para um maior aperfeiçoamento e reconhecimento das manisfestações clínicas das IST, de modo que esses acadêmicos se tornem profissionais capazes de diagnosticar e conduzir pacientes portadores de IST de forma correta. Além disso, em relação a comportamentos de risco, se faz necessária uma conscientização da população em estudo, de forma que apliquem os seus conhecimentos para auto prevenção de IST.

Recomenda-se que mais pesquisas semelhantes a essa sejam conduzidas, problematizando conhecimentos, comportamentos e adesão de acadêmicos frente a infecções sexualmente transmissíveis, principalmente em relação a fatores motivadores ao não uso da camisinha. Ademais, acredita-se que essa pesquisa fornece informações importantes que direcionam acadêmicos e profissionais na realização de práticas educativas voltadas para a população universitária.

\section{Referências}

Almeida, R. A. A. S., Corrêa, R. d. G. C. F., Rolim, I. L. T. P., Hora, J. M. d., Linard, A. G., Coutinho, N. P. S., \& Oliveira, P. d. S. (2017). Knowledge of adolescents regarding sexually transmitted infections and pregnancy. Revista Brasileira de Enfermagem, 70(5), 1033-1039. https://doi.org/10.1590/0034$\underline{7167-2016-0531}$

Aragão, J. C. S., Lopes, C. d. S., \& Bastos, F. I. (2011). Comportamento sexual de estudantes de um curso de medicina do Rio de Janeiro. Revista Brasileira de Educação Médica, 35(3), 334-340. https://doi.org/10.1590/s0100-55022011000300006

Araújo, T. M. E. d., \& Costa e Silva, N. d. (2014). Acidentes perfurocortantes e medidas preventivas para hepatite B adotadas por profissionais de Enfermagem nos serviços de urgência e emergência de Teresina, Piaú. Revista Brasileira de Saúde Ocupacional, 39(130), 175-183. https://doi.org/10.1590/0303-7657000079413

Brasil, Ministério da Saúde. (2020). Prevenção Combinada| Departamento de Doenças de Condições Crônicas e Infecções Sexualmente Transmissíveis. Home | Departamento de Doenças de Condições Crônicas e Infecções Sexualmente Transmissíveis. http://www.aids.gov.br/pt-br/publico-geral/previna-se

Brasil, Ministério da Saúde, Secretaria de Vigilância em Saúde Departamento de DST, Aids e. Hepatites Virais. (2015). Protocolo Clínico e Diretrizes Terapêuticas para Atenção Integral às Pessoas com Infecções Sexualmente Transmissíveis (2a ed.). Ministério da Saúde. https://bvsms.saude.gov.br/bvs/publicacoes/protocolo_clinico_diretrizes_terapeutica_atencao_integral_pessoas_infeccoes_sexualmente_transmissiveis.pdf

Fonte, V. R. F., Spindola, T., Francisco, M. T. R., Sodré, C. P., André. N. L. N. d. O \& Pinheiro, C. D. P. Jovens universitários e o conhecimento acerca das infecções sexualmente transmissíveis. Escola Anna Nery, 22 (2), 2018.

Da Fonte, V. R. F., Spindola, T., Lemos, A., Francisco, M. T. R., \& Oliveira, C. S. R. (2018). Conhecimento e percepção de risco em relação às infecções sexualmente transmissíveis entre jovens universitários. Cogitare Enfermagem, 23(3).

DE SALES, Jackeline Kérollen Duarte et al. Fatores de risco associados ao comportamento sexual de adolescentes. Revista Eletrônica Acervo Saúde, (49), e3382-e3382.

Gräf, D. D., Mesenburg, M. A \& Fassa, A. G (2020). Comportamento sexual de risco e fatores associados em universitários de uma cidade do sul do Brasil. Revista de Saúde Pública,

Guerra, F., Otaviano, R., Ramos, R., Damião, M \& Zanini, E., (2020). Comportamento sexual de estudantes universitários: um estudo de revisão. Fag Journal of Health. 2(2).

Henderson, J. T., Senger, C. A., Henninger, M., Bean, S. I., Redmond, N., \& O’Connor, E. A. (2020). Behavioral Counseling Interventions to Prevent Sexually Transmitted Infections. JAMA, 324(7), 682.

Jacobowski, B. (2010). Comportamento de Risco para HIV e DST entre Professores Universitários. Jornal Brasileiro de Doenças Sexualmente Transmissíveis, $199-205$.

Lima, I. B. (2018). Importância do diagnóstico precoce de HIV para a eficácia terapêutica e o bem-estar do paciente. Ces revista, $32(1)$, 15.

LINS, Laís Sandres et al. Análise do comportamento sexual de adolescentes. Revista Brasileira em Promoção da Saúde, 30(1). 
Research, Society and Development, v. 10, n. 15, e559101522003, 2021

(CC BY 4.0) | ISSN 2525-3409 | DOI: http://dx.doi.org/10.33448/rsd-v10i15.22003

Manoel, A. L., \& Trevisol, F. S. (2017). Comportamento sexual de estudantes de medicina do Brasil: um estudo multicêntrico. DST - J bras Doenças Sex Transm, 29(2), 6 .

Orlandi, Lara Evangelista; Rodrigues, Adriano; Garcia, Natália Galvão. Avaliação do conhecimento de jovens universitários quanto às infecções sexualmente transmissíveis. Research, Society and Development, 10(9), e31210918077-e31210918077, 2021.

Perim, L. F., Neutzling, A., Ventura, J \& Cabral, C. N. (2020). Fatores sócio eco culturais das infecções sexualmente transmissíveis: um enfoque na educação em saúdE. Research, Society and Development, 9(3), e115932140-e115932140, 2020.

Petrini, G. et al. (2014)Mudanças sociais e mudanças familiares. SEMOC-Semana de Mobilização Científica-Reforma Universitária Que Universidade o Brasil Quer?

Pimentel, M. H., Preto, L. S., Alves, M. J. G \& Monteiro, A. M. P. C. Comportamento sexual e estudantes do ensino superior. Psicologia, Saúde e Doenças. 17(3), 2016. 10.15309/16psd170304.

Ramos, R. C. d. A., Spindola, T., Oliveira, C. S. R., Martins, E. R. C., Lima, G. d. S. F., \& Araujo, A. S. d. B. d. (2020). Practices for the prevention of sexually transmitted infections among university students. Texto \& Contexto - Enfermagem, 29.

Rossi, L. F.d., Mazetto, F. M. C., Rezende, K. T. A., Marin, M. J. S., \& Nardo, L. R. d. O (2021). Educação em saúde relacionada a sexualidade e Infecções Sexualmente Transmissíveis: Uma revisão integrativa. New Trends in Qualitative Research, 8(1).

Spindola, T., Oliveira, C. S. R., Santana, R. S. C., Sodré, C. P., André, N. L. N. d. O., \& Brochado, E. D. J. (2019). Sexual Practices, Knowledge and Behavior of College Students Regarding Sexually Transmitted Diseases / Práticas Sexuais, Conhecimento e Comportamento dos Universitários em Relação às Infecções Sexualmente Transmissíveis. Revista de Pesquisa: Cuidado é Fundamental Online, 11(5), 1135.

Wanderley, M. d. S., Sobral, D. T., Levino, L. d. A., Marques, L. d. A., Feijó, M. S., \& Aragão, N. R. C. (2019). Students' HPV vaccination rates are associated with demographics, sexuality, and source of advice but not level of study in medical school. Revista do Instituto de Medicina Tropical de São Paulo, 61

Zardo, G. P., Farah, F. P., Mendes, F. G., Franco, C. A. G. d. S., Molina, G. V. M., Melo, G. N. d., \& Kusma, S. Z. (2014). Vacina como agente de imunização contra o HPV. Ciência \& Saúde Coletiva, 19(9), 3799-3808. 